\title{
The association between postoperative corneal edema and phacoemulsification level
}

\author{
Enes Uyar \\ Department of Ophthalmology, Aksaray Training and Research Hospital, Aksaray University, Aksaray, Turkey
}

\section{ABSTRACT}

\begin{abstract}
Aim: To evaluate the effect of phacoemulsification level on postoperative central corneal thickness (CCT) differences and to assess the effects of demographic characteristics, biometric values and surgical parameters of patients on postoperative corneal edema.

Methods: This prospective study included 232 eyes of 232 patients who underwent phacoemulsification surgery. Biometric values, phacoemulsification time, phacoemulsification power and effective phacoemulsification time (EPT) were evaluated. CCT measurements were repeated at follow-ups on days 1,7 and 30 after surgery. Phaco-chop technique was performed in all patients and phacoemulsification level changes that occurred during surgery were recorded. Patients were grouped as Group 1: >75\% of lens nucleus emulsified in capsular bag; Group 2: >75\% of lens nucleus emulsified in iris plane; and Group 3: > $50 \%$ of lens nucleus emulsified in anterior chamber.
\end{abstract}

Results: Increase in the mean CCT values on the first day (incCCT1) and at the first week after surgery were more pronounced in Group 3 as compared with both Groups 1 and 2 ( $p<0.05$ for all). In addition, incCCT1 was higher in Group 2 than Group $1(p=0.040)$. In the final model of multiple linear regression $\left(\mathrm{R}^{2}=0.31\right)$, it was determined that EPT, aqueous depth (AD) and phacoemulsification level significantly affected incCCT1 (p range: $<0.001$ to 0.009 ).

Conclusions: The present study showed that more anterior phacoemulsification levels than the capsular bag caused higher CCT increase postoperatively. In addition, AD, EPT and phacoemulsification level were found as the most significant factors that influence postoperative corneal edema.

Key words: Central corneal thickness, corneal edema, phacoemulsification level, surgery.

$\triangle$ Dr. Enes Uyar

Department of Ophthalmology, Aksaray Training and Research Hospital, Aksaray University, Aksaray, Turkey E-mail: enuyar@gmail.com

Received: 2021-10-26/Revisions: 2021-11-24

Accepted: 2021-12-04 / Published online: 2022-01-01

\section{Introduction}

Currently, phacoemulsification surgery is being routinely used to treat cataract [1-3]. Although this is a safe and effective procedure, ultrasound energy used during the surgery can damage the corneal endothelium, and endothelial cell loss (ECL) can cause corneal edema [4-6]. Although corneal edema is generally transient and mild, it cannot be recovered in some cases involving excessive ECL [6-8]. Several factors that may affect postoperative corneal edema have been proposed such as phacoemulsification time, manipulation of devices, nucleus hardness, surgery technique, surgeon experience, fluid and vacuum dynamics, Descemet's membrane detachment, and baseline endothelial cell count [5-7,9]. Another factor that may affect postoperative corneal edema and ECL is the depth of phaco probe in the anterior segment while the lens nucleus is emulsified. This factor 
has been termed as "phacoemulsification level" in this study. When phacoemulsification level approaches the endothelium, the harmful effect of ultrasound energy on the endothelium may increase $[5,10,11]$. Previous studies that did not use the ophthalmic viscoelastic device (OVD) demonstrated higher ECL in anterior chamber phacoemulsification $[12,13]$. Koch et al. [14] have also found that iris plane phacoemulsification causes more ECL than the posterior chamber phacoemulsification even if OVD is used. In contrast, there is no considerable difference in postoperative central corneal thickness (CCT) between the pure posterior chamber techniques and some supracapsular techniques that involve more maneuvers and phacoemulsification in the anterior chamber $[4,15]$.

The surgical technique during the phacoemulsification procedure may require modification owing to several factors such as patient compliance, surgeon comfort, intraoperative pupil changes, anterior chamber dynamics, diameter of the capsulorhexis, prolapses of the lens to the anterior chamber during hydrodissection, catch of the nuclear fragments by OVD in the anterior chamber, avoiding posterior capsule rupture, and surgeon experience. Therefore, phacoemulsification level may change during surgery when the same surgical technique is use. The present study aimed to determine the effect of phacoemulsification level changes occurring during the surgery on postoperative CCT differences. In addition, the effect of demographic characteristics, biometric values, and surgical parameters of patients on postoperative corneal edema was evaluated.

\section{Materials and methods}

The present study was designed on a prospective pattern, and it was performed on
232 consecutive patients who were operated in Ophthalmology Clinic between October 3, 2019 and December 31, 2020. Informed consent was obtained from all participants. The study was conducted with the approval of Aksaray University Ethical Committee in accordance with the Helsinki Declaration (Protocol number: 2019/10-02).

All patients underwent complete ophthalmologic examinations preoperatively, including best corrected visual acuity assessment, slit-lamp and fundus examinations, and intraocular pressure (IOP) measurement. Nuclear sclerosis was evaluated clinically according to the Lens Opacities Classification System 3. Biometric parameters, aqueous depth (AD), axial length (AL), lens thickness (LT), and pupil diameter measurements after full dilatation were evaluated using a lowcoherence optical biometry device (Haag-Strait Diagnostics Biometer LS-900; Haag-Strait AG, Switzerland). Scheimpflug imaging instrument (Sirius, Costruzione Strumenti Oftalmici, and Florence, Italy) was used to evaluate CCT. Ophthalmologic examinations, CCT, and IOP measurements were repeated after surgery on day 1,7, and 30. Measurements were performed by an experienced examiner who was blinded to the surgical data of patients.

The inclusion criteria were as follows: NO3 $\mathrm{NC} 3$ or NO4 NC4 grade senile cataract according to LOCS scale, best corrected visual acuity of lower than 10/20 with the decimal notation, age above 40 years, not having an acute or chronic eye disease (glaucoma, uveitis, diabetic retinopathy, etc.) and no previous ophthalmological surgery history. The exclusion criteria were as follows: preexisting corneal pathology, i.e. cornea guttata, corneal dystrophy, corneal degenerations and other corneal inflammations, pseudoexfoliation syndrome, using iris hooks during surgery 
because of small pupil and any eye pathology preventing preoperative and postoperative measurements. Patients who developed intraoperative complications were also excluded from the statistical analysis.

The same surgeon (EU) performed all operations using the same phacoemulsification machine (OS4, Oertli Instrumente AG, Berneck, Switzerland). Preoperatively, tropicamide $1 \%$, cyclopentolate hydrochloride $1.0 \%$, and phenylephrine hydrochloride $2.5 \%$ eye drops were applied to ensure full pupil dilatation. Before the operation, proparacaine hydrochloride $0.5 \%$ eye drops were topically administered for topical anesthesia. After 5\% povidone iodine solution was instilled to the conjunctival sac, the periocular skin was cleaned with $10 \%$ povidone iodine. The eye was covered with a sterile drape and opened with a lid speculum. Paracenteses were performed both temporally and nasally, and sodium hyaluronate $2.0 \%$ (Protectalon ${ }^{\circledR}$ ) OVD was injected into the anterior chamber. Clear corneal main incision was made with a $2.75 \mathrm{~mm}$ blade at 11 o'clock position. Then, a continuous curvilinear capsulorhexis (approximately 5.5 $6 \mathrm{~mm}$ diameter) and hydrodissection were completed. After the central cortical and epinuclear materials were removed, the phaco tip was buried with burst energy mode $(50 \%$ cycle, $30 \mathrm{msec}$ length) into the superior part of nucleus, and occlusion was performed under a high vacuum setting $(400 \mathrm{mmHg})$. The bottle height was $90 \mathrm{~cm}$. A chopper was placed underneath the anterior capsule at 6 o'clock position and moved through the phaco tip to crack the nucleus initially. After the first crack, the chopper and phaco tip were moved in opposite directions to separate the nuclear halves. Then, the nucleus was rotated, and half of it was impaled by the phaco tip. Chopping was performed, and the first free nuclear piece was emulsified. This process was repeated under the same energy and vacuum settings in the capsular bag as much as possible until the nuclear material was completely consumed. If the moving lens fragments were emulsified closer to the anterior chamber than the capsular bag, the changes in phacoemulsification level were recorded. Patients were classified into three groups according to the percentage of lens nucleus emulsified at a particular phacoemulsification level (Table 1). Patients who did not meet any of the group criteria were excluded from the study.

Table 1. Group classification according to phacoemulsification level.

\begin{tabular}{|l|l|}
\hline Group 1 & $\begin{array}{l}75 \%-100 \% \text { of the lens nucleus } \\
\text { was emulsified in the bag, 0\%- } \\
25 \% \text { of the lens nucleus was } \\
\text { emulsified in the iris plane. }\end{array}$ \\
\hline Group 2 & $\begin{array}{l}75 \%-100 \% \text { of the lens nucleus } \\
\text { was emulsified in the iris plane, } \\
0 \%-25 \% \text { of the lens nucleus was } \\
\text { emulsified in the anterior } \\
\text { chamber or in the bag. }\end{array}$ \\
\hline Group 3 & $\begin{array}{l}50 \%-75 \% \text { of the lens nucleus } \\
\text { was emulsified in the anterior } \\
\text { chamber, 25\%-50\% of the lens } \\
\text { nucleus was emulsified in the iris } \\
\text { plane. }\end{array}$ \\
\hline
\end{tabular}

Epinucleus was cleared with low vacuum and ultrasound power settings. Cortex removal was performed by bimanual irrigation/aspiration. After all lens materials were cleared, sodium hyaluronate $1.4 \%$ (Protectalon ${ }^{\circledR}$ ) OVD was injected into the capsular bag. Same foldable one-piece hydrophobic acrylic intraocular lens (Sensar AR40, AMO, Mineapolis, USA) was inserted to each patient. Then OVD was removed from anterior and posterior chamber 
and corneal incisions were closed by stromal hydration without any suturing. At the end of surgery, intracameral cefuroxime $1 \mathrm{mg} / 0.1 \mathrm{ml}$ was applied and corneal incisions were checked in order to ensure that there was no leakage.

The phacoemulsification time (s), phacoemulsification power (\%), and effective phacoemulsification time (EPT) were recorded. EPT was computed as follows: phacoemulsification time $\times$ phacoemulsification power/100. Total operation time was calculated as the time between the opening of the first paracentesis and the closure of corneal incisions by stromal hydration. Postoperatively, patients were treated with moxifloxacin $0.5 \%$ and dexamethasone $0.1 \%$ eye drops 6 times daily for 1 week, after which only dexamethasone $0.1 \%$ was instilled over 3 weeks with a gradually decreasing dosage.

\section{Statistical analyses}

All data was analyzed using the SPSS statistical software package, version 24.0 (SPSS Inc., Chicago, IL, USA). Parametric data was compared using analysis of variance (ANOVA), followed by a Tukey's honestly significant difference test for post hoc comparisons, and non-parametric data was compared using the chi-square test. The associations with continuous and categorical variables were assessed using Pearson's and Spearman's-Rho bivariate correlation analyses, respectively. The factors that could affect the central corneal edema on the postoperative first day were evaluated using multiple linear regression analysis. The statistical significance level was set at $\mathrm{p}<0.05$. Normal distribution of the data was checked by Kolmogorov-Smirnov test. For all of the studied parameters, the $p$ value for the Kolmogorov-Smirnov test was $>0.05$.

\section{Results}

In total, 232 eyes of 232 patients were included in this study. Of these patients, 129 were male (55.6\%) and 103 were female (44.4\%), with an average age of $69.8 \pm 7.4$ years. Of the 232 eyes, 107 (46.1\%) were right and 125 (53.9\%) were left. Overall, 104 patients had NO3 NC3 $(44.8 \%)$ and 128 patients had NO4 NC4 $(55.2 \%)$ cataract. There were 87 patients (37.5\%) in Group 1, 79 patients $(34.1 \%)$ in Group 2, and 66 patients (28.4\%) in Group 3. Regarding intraoperative complications, two cases of posterior capsule ruptures without vitreous loss, one of zonular dehiscence, six of small detachments of Descemet's membrane, and two of radial capsulorhexis tears were noted. No postoperative complication was observed during the follow-up.

Biometric and surgical data of patients are provided in Table 2. The mean values of CCT along with mean differences between preoperative CCT (preCCT) and postoperative CCT values of groups are shown in Table 3. There was no difference among the groups in IOP values as well as among IOP differences on days 1,7 , and 30 after surgery ( $p>0.05$ for all). Biometric and surgical data were similar in all groups ( $p>0.05$ for all) (Table 2). There were statistically significant differences between groups in postoperative CCT values on the first day (postCCT1) and postoperative CCT values at the first week ( $p<0.001$ for both). Postoperative CCT values in the first month were not different among the groups ( $p=0.352$ ) (Table 3).

In post hoc comparisons, an increase in the mean CCT values after surgery on the first day and at the first week were more pronounced in Group 3 than in Group 1 and Group 2 ( $p<0.05$ for all). One month after the surgery, these differences were not statistically significant ( $p$ $>0.05$ for all). In addition, an increase in the 
Table 2. Biometric and surgical parameters of the groups.

\begin{tabular}{|c|c|c|c|c|c|}
\hline \multicolumn{2}{|c|}{ Parameters } & $\begin{array}{c}\text { Group 1 } \\
(\mathbf{n}=87)\end{array}$ & $\begin{array}{c}\text { Group } 2 \\
(n=79)\end{array}$ & $\begin{array}{c}\text { Group } 3 \\
(n=66)\end{array}$ & $P$ \\
\hline \multicolumn{2}{|c|}{ Age (years) } & $69.1 \pm 7.7$ & $71.0 \pm 7.2$ & $69.9 \pm 6.9$ & $0.284 *$ \\
\hline \multicolumn{2}{|c|}{ EPT (sec) } & $3.8 \pm 2.2$ & $3.8 \pm 1.5$ & $3.9 \pm 1.5$ & $0.992 *$ \\
\hline \multicolumn{2}{|c|}{ Phaco time (sec) } & $14.0 \pm 7.5$ & $14.1 \pm 5.7$ & $14.8 \pm 5.1$ & $0.862 *$ \\
\hline \multicolumn{2}{|c|}{ Phaco power (\%) } & $28.1 \pm 4.9$ & $28.2 \pm 5.6$ & $26.3 \pm 4.7$ & $0.213 *$ \\
\hline \multicolumn{2}{|c|}{ Total operation time (min) } & $13.5 \pm 2.9$ & $12.8 \pm 2.8$ & $12.7 \pm 2.3$ & $0.269 *$ \\
\hline \multicolumn{2}{|c|}{ Preoperative IOP (mmHg) } & $14.1 \pm 2.8$ & $14.5 \pm 3.7$ & $13.7 \pm 3.0$ & $0.436^{*}$ \\
\hline \multicolumn{2}{|c|}{$\mathbf{A L}(\mathbf{m m})$} & $23.5 \pm 0.8$ & $23.5 \pm 0.9$ & $23.6 \pm 1.0$ & $0.869 *$ \\
\hline \multicolumn{2}{|c|}{$\mathrm{AD}(\mathbf{m m})$} & $2.8 \pm 0.4$ & $2.9 \pm 0.4$ & $2.9 \pm 0.3$ & $0.422 *$ \\
\hline \multicolumn{2}{|c|}{$\mathbf{L T}(\mathbf{m m})$} & $4.4 \pm 0.4$ & $4.3 \pm 0.4$ & $4.4 \pm 0.5$ & $0.817 *$ \\
\hline \multicolumn{2}{|c|}{ PD (mm) } & $6.9 \pm 1.0$ & $6.8 \pm 0.9$ & $7.0 \pm 1.0$ & $0.600 *$ \\
\hline \multirow[b]{2}{*}{ NS } & Grade 3 n=126 & 50 & 39 & 37 & \multirow[b]{2}{*}{$0.494 * *$} \\
\hline & $\begin{array}{l}\text { Grade } 4 \\
\text { n=106 }\end{array}$ & 37 & 40 & 29 & \\
\hline \multicolumn{6}{|c|}{ AD: Aqueous depth, AL: Axial length, EPT: Effective phacoemulsification time, LT: Lens thickness, NS } \\
\hline
\end{tabular}

mean CCT values on the postoperative first day (incCCT1) was higher in Group 2 than in Group $1(p=0.040)$.

In the correlation analyses, the factors that could be associated with incCCT1 were as follows: age, sex, EPT, AD, phacoemulsification level, phacoemulsification time, and phacoemulsification power ( $r$ range: -0.246 to 0.420 , $p$ range: $<0.001-0.027)$. No significant relationship was noted among NS grade, AL, pupil diameter, total operation time, LT, and incCCT1 ( $r$ range: -0.121 to $0.131, p$ range: 0.093-0.950). A multiple linear regression analysis, in which all independent variables were entered into the model simultaneously, was conducted to define independent factors associated with incCCT1. The first model was developed with factors that were associated with postoperative ECL or corneal edema in previous studies $[5,6,9,16]$. Next, phacoemulsification level was added into the model. The final multiple linear regression model indicated that EPT, AD, and phacoemulsification level significantly affected incCCT1. The model explained $31 \%$ of the variation in incCCT1 and revealed that the effect of phacoemulsification level accounted for $13 \%$.

\section{Discussion}

The present study reported that the increase in postoperative CCT values was higher in the anterior chamber phacoemulsification than in the posterior chamber phacoemulsification or the iris plane phacoemulsification. The effect of the anterior chamber phacoemulsification 
Table 3. CCT values and postoperative CCT differences of the groups.

\begin{tabular}{|c|c|c|c|c|}
\hline Parameters & $\begin{array}{c}\text { Group } 1 \\
(\mathrm{n}=87)\end{array}$ & $\begin{array}{c}\text { Group } 2 \\
(n=79)\end{array}$ & $\begin{array}{c}\text { Group } 3 \\
(n=66)\end{array}$ & $\boldsymbol{P}$ \\
\hline $\operatorname{PreCCT}(\mu \mathrm{m})$ & $531.5 \pm 27.4$ & $534.3 \pm 29.0$ & $540.2 \pm 25.3$ & $0.322 *$ \\
\hline PostCCT1 $(\mu \mathrm{m})$ & $573.7 \pm 35.6$ & $594.7 \pm 45.4$ & $627.2 \pm 56.3$ & $<0.001 *$ \\
\hline PostCCT7 $(\mu \mathrm{m})$ & $545.8 \pm 33.8$ & $545.0 \pm 32.9$ & $576.6 \pm 45.9$ & $<0.001 *$ \\
\hline PostCCT30 $(\mu \mathrm{m})$ & $533.1 \pm 27.2$ & $539.8 \pm 29.7$ & $544.2 \pm 25.2$ & $0.352 *$ \\
\hline PostCCT1-PreCCT $(\mu \mathrm{m})$ & $42.2 \pm 17.3$ & $60.4 \pm 32.7$ & $81.4 \pm 50.3$ & $<0.001 *$ \\
\hline PostCCT7-PreCCT $(\mu \mathrm{m})$ & $12.0 \pm 11.5$ & $14.2 \pm 13.3$ & $29.9 \pm 28.4$ & $<0.001 *$ \\
\hline PostCCT30-PreCCT $(\mu \mathrm{m})$ & $1.1 \pm 2.9$ & $1.4 \pm 2.8$ & $3.0 \pm 5.0$ & $0.181^{*}$ \\
\hline $\begin{array}{l}\text { Percentage of } \\
(\text { PostCCT1-PreCCT) / PreCCT (\%) }\end{array}$ & $7.9 \pm 3.2$ & $11.3 \pm 6.2$ & $15.3 \pm 9.6$ & $<0.001 *$ \\
\hline $\begin{array}{l}\text { Percentage of } \\
(\text { PostCCT7-PreCCT) / PreCCT }(\%)\end{array}$ & $2.3 \pm 2.2$ & $2.7 \pm 2.5$ & $5.5 \pm 5.1$ & $0.002 *$ \\
\hline $\begin{array}{l}\text { Percentage of } \\
(\text { PostCCT30-PreCCT) / PreCCT }(\%)\end{array}$ & $0.2 \pm 0.6$ & $0.3 \pm 0.5$ & $0.5 \pm 0.9$ & $0.198^{*}$ \\
\hline
\end{tabular}

persisted for more than a week. In addition, AD, EPT, and phacoemulsification level were factors significantly affecting incCCT1.

Recent studies have revealed that anterior chamber phacoemulsification or supracapsular techniques can be used safely and have certain advantages [4,15,17-19]. Hwang et al. [4] compared the retro-chop and stop-and-chop techniques according to the postoperative ECL and CCT increase. In the retro-chop group, phacoemulsification was performed in the iris plane, which resulted in lower ECL than in the stop-and-chop group. The authors have proposed that reduced phacoemulsification time used in the retro-chop group caused less ECL. In addition, Alio et al. [17] found no difference in postoperative CCT and ECL between the stop-and-chop and the phaco-out groups, in which phacoemulsification was performed entirely in the anterior chamber. The authors have attributed these findings in the phaco-out group to the use of copious dispersive OVD [17]. However, clinical examinations revealed that the corneal edema was more pronounced in the phaco-out group during the postoperative first 2 weeks [17]. In the half-moon technique used by Can et al.[18], the lens nucleus was prolapsed at the supracapsular level at the start of the operation; after the first crack, it was pushed back into the capsular bag. The authors suggested that the half-moon technique can be used as safely as posterior phacoemulsification strategies. However, the CCT values on the first day after surgery were higher in the half-moon group, even though EPT was less than the stop-andchop group. The authors attributed this difference to the fact that chopping was 
performed closer to the corneal endothelium at the beginning of the surgery [18]. In contrast, Kosrirukvongs et al. [20] found that the ECL percentage in postoperative first week was significantly higher in the chip-and-flip group (21\%) than in the divide-and-conquer group (12.6\%). The authors did not detect a difference in mean postoperative CCT values between two groups; however, CCT values on the postoperative first day were not evaluated in this study. The authors specified that more surgical trauma occurred in the chip-and-flip group, even though OVD was used to protect the corneal endothelium [20]. Likewise, Koch et al. [14] deduced that iris plane phacoemulsification caused more endothelial injury than posterior chamber phacoemulsification, particularly when cohesive OVD was used instead of dispersive OVD. Although different phacoemulsification techniques have been thoroughly compared in above mentioned studies, the present study assessed the effect of possible phacoemulsification level changes on postoperative corneal edema while the same technique is used. The phaco-chop technique was used for nuclear fragmentation in all patients, and we observed that phacoemulsification performed at levels closer to the anterior chamber than the capsular bag lead to more corneal edema. In regression analyses, the phacoemulsification level of Group 3 had the most significant effect on inducing incCCT1, whereas that of Group 1 resulted in significantly reduced incCCT1.

In previous studies that investigated similar preoperative and intraoperative factors, phacoemulsification time, phacoemulsification power, age, AL, NS grade, pupil diameter, total infusion volume, implanted IOL type, surgeon experience and total operation time were found to be determinants of postoperative corneal edema $[3,5,6,9,16,21]$. In this study, however, we detected that AL did not have a remarkable effect on corneal edema whereas $\mathrm{AD}$ values were negatively correlated with postoperative CCT increase. Several studies suggested that the phacoemulsification closer to corneal endothelium might have much harmful effect on cornea, however no significant association between $\mathrm{AD}$ and postoperative ECL was found $[5,11,16]$. Walkow et al. [5] have explained their results by the deepening of anterior chamber during surgery. O'brien et al. [16] have attributed the lack of association between shallow anterior chamber and ECL to the contribution of reduced wound leakage during surgery. This difference might be due to the non-use of dispersive OVD which caused the effect of shallow AD on corneal edema to become more pronounced [11,14,22]. Another reason of dissimilar results might be the differences in bottle heights or total infusion volumes.

Many studies have reported an association between phacoemulsification time and postoperative corneal edema or ECL $[5,6,16,21,23,24]$.

Likewise, phacoemulsification time and EPT were significantly related with incCCT1 in the present study. Several mechanisms have been proposed how ultrasound energy may damage corneal endothelium [23,25,26]. Moreover, it has been shown that NS grade might affect corneal edema by increasing EPT [5,9]. In the present study, the absence of a significant relationship between LT or NS grade and incCCT1 may be caused by the inclusion of only grade NO3 NC3 and NO4 NC4 cataracts. The literature may have possible explanations for the different results observed regarding the factors affecting affect corneal edema. Firstly, there were differences in measurement devices, evaluated postoperative parameters, surgery 
techniques, phaco machines, OVD types, surgeon experience, and methods used in the studies. Additionally, many intraoperative dynamics such as bottle height, total infusion volume, and wound leakage might yield different results in studies that evaluated how preoperative biometric parameters of patients affect the corneal damage. Especially, the type and amount of OVD used during surgery might affect the relationship between AD, AL, EPT, NS grade, and postoperative corneal edema or ECL. The regression model of the present study explained $31 \%$ of the variation in incCCT1; thus, there may be other factors influencing incCCT1, which could not be identified by regression models. These factors include OVD types, baseline endothelium cell count, surgeon experience, total infusion volume, and phaco tip position [3,5,6,9,11,16,22-24].

The present study possesses the following limitations: CCT values could have been measured in earlier hours postoperatively and follow-ups could have been arranged more frequently. The durations of surgery steps were not calculated separately. The surgeon observed the percentage of emulsified lens nucleus during surgery to decide the phacoemulsification level. This method can be criticized as not being objective enough. Few possible predictors of the postoperative corneal edema such as total infusion volume, the amount of OVD used, the position of phaco tip, and baseline endothelium cell count were not evaluated. One more limitation of the study is that only CCT measurements were used to evaluate the effect of phacoemulsification procedure on the corneal edema, and postoperative ECL was not assessed. However, previous studies have suggested that CCT measurements have greater availability, accuracy, and importance clinically to reveal endothelial damage [6,18]. In addition, a significant correlation has already been observed between the increase in CCT and ECL after surgery $[8,27,28]$. In contrast, the present study has certain advantages. The effect of phacoemulsification level on the cornea was evaluated objectively with the contribution of standardized variables, i.e., nuclear fragmentation technique, NS grade, used OVD, surgeon, and phaco machine. In addition to these, as the phacoemulsification time, phacoemulsification power, demographic, and biometric parameters of the groups were similar, the effect of phacoemulsification level could be evaluated independently. Furthermore, the study method could adequately represent cataract surgery practice, because phacoemulsification level changes were evaluated during live surgery.

\section{Conclusion}

In conclusion, the current study showed that phacoemulsification levels closer to the anterior chamber than the capsular bag resulted in more negative effect on the cornea. Moreover, this effect might continue longer than 1 week. Thus, phacoemulsification in the capsular bag may be safer for the corneal endothelium and can still be a primary choice in routine cataract cases. At the same time, advances in modern surgical techniques and instruments have made supracapsular techniques, which have a relatively shorter learning curve, more preferable, especially in cases such as zonular dehiscence, lens subluxation, hard cataracts with posterior plate, small pupil, and advanced pseudoexfoliation. However, if more than half of the lens nucleus is to be emulsified in the anterior chamber, abundant use of dispersive OVD is strongly recommended. 
Funding: The author(s) received no financial support for the research, authorship, and/or publication of this article.

Conflict of Interest: The authors declare that they have no conflict of interest.

Ethical statement: The study was conducted with the approval of Aksaray University Ethical Committee in accordance with the Helsinki Declaration (Protocol number: 2019/10-02).

\section{Open Access Statement}

This is an open access journal which means that all content is freely available without charge to the user or his/her institution under the terms of the Creative Commons Attribution NonCommercial License (https://creativecommons.org/licenses/by/4.0/). Users are allowed to read, download, copy, distribute, print, search, or link to the full texts of the articles, without asking prior permission from the publisher or the author.

Copyright (c) 2021: Author (s).

\section{References}

[1] Linebarger EJ, Hardten DR, Shah GK, et al. Phacoemulsification and modern cataract surgery. Surv Ophthalmol. 1999;44(2):12347.

[2] Olson RJ, Mamalis N, Werner L, et al. Cataract treatment in the beginning of the 21st century. Am J Ophthalmol. 2003;136(1):146-54.

[3] Wong T, Hingorani M, Lee V. Phacoemulsification time and power requirements in phaco chop and divide and conquer nucleofractis techniques. J Cataract Refract Surg. 2000;26(9):1374-78.

[4] Hwang HS, Lee JH, Jeon SJ, et al. StopChop versus Retro-Chop Nucleotomy During Phacoemulsification. Curr Eye Res. 2016;41(11):1454-59.

[5] Walkow T, Anders N, Klebe S. Endothelial cell loss after phacoemulsification: relation to preoperative and intraoperative parameters. J Cataract Refract Surg. 2000;26(5):727-32.

[6] Lhuillier L, Jeancolas AL, Renaudin L, et al. Impact of Ophthalmic Surgeon Experience on Early Postoperative Central Corneal Thickness After Cataract Surgery. Cornea. 2017;36(5):541-45.

[7] Sharma N, Singhal D, Nair SP, et al. Corneal edema after phacoemulsification. Indian $\mathrm{J}$ Ophthalmol. 2017;65(12):1381-89.

[8] Lundberg B, Jonsson M, Behndig A. Postoperative corneal swelling correlates strongly to corneal endothelial cell loss after phacoemulsification cataract surgery. Am J Ophthalmol. 2005;139(6):1035-41.

[9] Hayashi K, Hayashi H, Nakao F, et al. Risk factors for corneal endothelial injury during phacoemulsification. J Cataract Refract Surg. 1996;22(8):1079-84.

[10]De Bernardo M, Rosa N. Central corneal thickness measurement after cataract surgery. Cornea. 2018;37(4):e19-e20.

[11]Pereira ACA, Porfírio Jr F, Freitas LL, et al. Ultrasound energy and endothelial cell loss with stop-and-chop and nuclear preslice phacoemulsification. J Cataract Refract Surg. 2006;32(10):1661-66.

[12]Kraff MC, Sanders DR, Lieberman HL. Specular microscopy in cataract and intraocular lens patients: a report of 564 cases. Arch Ophthalmol. 1980;98(10):178284.

[13] Sugar J, Mitchelson J, Kraff M. The effect of phacoemulsification on corneal endothelial cell density. Arch Ophthalmol. 1978;96(3):446-48.

[14] Koch DD, Liu JF, Glasser DB, et al. A comparison of corneal endothelial changes after use of Healon or Viscoat during phacoemulsification. Am J Ophthalmol. 1993;115(2):188-201. 
[15] Jeancolas A-L, Lhuillier L, Renaudin L, et al. Central corneal thickness assessment after phacoemulsification: Subluxation versus Divide-and-Conquer. $\mathrm{J} \quad \mathrm{Fr}$ d'Ophtalmol. 2017;40(9):744-50.

[16]D O'Brien P, Fitzpatrick P, Kilmartin DJ, et al. Risk factors for endothelial cell loss after phacoemulsification surgery by a junior resident. J Cataract Refract Surg. 2004;30(4):839-43.

[17]Alió JL, Mulet ME, Shalaby AM, Attia WH. Phacoemulsification in the anterior chamber. J Cataract Refract Surg. 2002;28(1):67-75.

[18]Can İ, Takmaz T, Genç İ. Half-moon supracapsular nucleofractis phacoemulsification: safety, efficacy, and functionality. J Cataract Refract Surg. 2008;34(11):1958-65.

[19] Gross FJ, Garcia-Zalisnak DE, Bovee CE, et al. A comparison of pop and chop to divide and conquer in resident cataract surgery. Clinical ophthalmology. 2016;10:1847-51.

[20]20. Kosrirukvongs P, Slade SG, Berkeley RG. Corneal endothelial changes after divide and conquer versus chip and flip phacoemulsification. J Cataract Refract Surg. 1997;23(7):1006-12.

[21]Dick HB, Kohnen T, Jacobi FK, et al. Longterm endothelial cell loss following phacoemulsification through a temporal clear corneal incision. J Cataract Refract Surg. 1996;22(1):63-71.

[22]Behndig A, Lundberg B. Transient corneal edema after phacoemulsification: comparison of 3 viscoelastic regimens. J Cataract Refract Surg.. 2002;28(9):1551-56.

[23] Faramarzi A, Javadi MA, Karimian F, et al. Corneal endothelial cell loss during phacoemulsification: bevel-up versus beveldown phaco tip. J Cataract Refract Surg. 2011;37(11):1971-76.
[24]Pirazzoli G, D'Eliseo D, Ziosi M, et al. Effects of phacoemulsification time on the corneal endothelium using phacofracture and phaco chop techniques. J Cataract Refract Surg. 1996;22(7):967-69.

[25] Packer M, Fishkind WJ, Fine IH, et al. The physics of phaco: a review. J Cataract Refract Surg. 2005;31(2):424-31.

[26]Raskin E, Paula JS, Cruz AAV, et al. Effect of bevel position on the corneal endothelium after phacoemulsification. Arquivos brasileiros de oftalmologia. 2010;73(6):50810.

[27]Cheng H, Bates AK, Wood L, et al. Positive correlation of corneal thickness and endothelial cell loss: serial measurements after cataract surgery. Archives of ophthalmology. 1988;106(7):920-22.

[28] Perone JM, Boiche M, Lhuillier L, et al. Correlation between postoperative central corneal thickness and endothelial damage after cataract surgery by phacoemulsification. Cornea. 2018;37(5):587-90. 